\title{
Proximal fibular resections for primary bone tumours: oncological and functional results of a case series
}

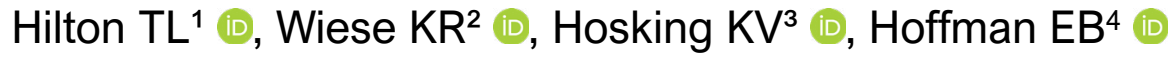 \\ $1 \mathrm{MBChB}(\mathrm{UCT}), \mathrm{DA}(\mathrm{SA})$, DipPEC(SA), FCOrth(SA), MMed(UCT); Orthopaedic Consultant, Groote Schuur \& Vincent Pallotti Hospitals, Cape Town, \\ University of Cape Town, South Africa \\ $2 \mathrm{MBChB}$ (UCT), Orthopaedic Registrar, Division of Orthopaedic Surgery, Department of Surgical Sciences, Faculty of Medicine and Health Sciences, \\ Stellenbosch University, South Africa \\ $3 \mathrm{MBChB}(\mathrm{UCT})$, FCOrth(SA), Orthopaedic Consultant, Vincent Pallotti Life Orthopaedic Hospital, Cape Town, University of Cape Town, South Africa \\ 4 MBChB(US), FCS Orth(SA), Emeritus Associate Professor, University of Cape Town, South Africa
}

Corresponding author: Dr TL Hilton, 11 Clive Street, Vredehoek, Cape Town, 8000; Email: thilton@hotmail.com; cell phone: 0027 (82) 796 7608

\begin{abstract}
Background: Resection of aggressive benign or malignant tumours of the proximal fibula are difficult due to the high number of surrounding anatomical compartments and close association with many important neurovascular and functional structures. For the same reasons malignant tumours behave differently in this area. Before the 1980s results were poor. With the introduction of neoadjuvant chemotherapy and limb salvage surgery, Malawer described a technique of local en bloc resection. This study presents the oncological and functional results of a case series using this technique.
\end{abstract}

Patients and methods: A retrospective folder review of 14 patients was done. Six patients with a large active, an aggressive benign or a low-grade malignant tumour had a Malawer type I marginal resection, and eight patients with a stage IIB malignant sarcoma had a Malawer type II wide intracompartmental resection sacrificing the common peroneal nerve. The follow-up at a median of 38 months included the imaging, histology and a functional MSTS score.

Results: The median age of the type I resections was 42.5 years; giant cell tumour was the commonest tumour (50\%); and the median functional MSTS score at follow-up was 29. The median age of the type II resections was 12 years; osteoblastic osteosarcoma was the commonest tumour (75\%); and the median functional MSTS score was 26 . All type II resections achieved clear margins at the initial surgery and there were no recurrences or metastases in either group. There were no wound complications and no vascular complications in spite of sacrificing the tibialis anterior artery in some type I and all type II resections, and the peroneal artery in type II resections. No patient complained of knee instability. The main functional impairment was due to loss of common peroneal function which required an AFO in some patients and a tibialis posterior tendon transfer in one patient.

Conclusions: Resection of benign and malignant tumours of the proximal fibula achieved good cure rates and functional results, despite the sacrifice of the common peroneal nerve.

Level of evidence: Level 4

Key words: benign aggressive and malignant primary bone tumours, proximal fibula, Malawer procedure

Citation: Hilton TL, Wiese KR, Hosking KV, Hoffman EB. Proximal fibular resections for primary bone tumours: oncological and functional results of a case series. SA Orthop J 2019;18(2):44-48. http://dx.doi.org/10.17159/2309-8309/2019/v18n2a5

Editor: Prof TLB le Roux, University of Pretoria, South Africa

Received: October 2018

Accepted: March 2019

Published: May 2019

Copyright: ( 2019 Hilton TL, et al. This is an open-access article distributed under the terms of the Creative Commons Attribution Licence, which permits unrestricted use, distribution and reproduction in any medium, provided the original author and source are credited.

Funding: No external funding was received for this study.

Conflict of interest: The authors declare they have no conflicts of interest that are directly or indirectly related to the research. 


\section{Introduction}

Primary bone tumours of the proximal fibula are rare, comprising only $2.5 \%$ of bone tumours. ${ }^{1}$ Approximately half of these tumours are malignant. ${ }^{1}$ Abdel et al. ${ }^{2}$ found an incidence of malignant tumours of the proximal fibula of 1.2 per year (112 tumours at the Mayo Clinic from 1910 to 2007).

Although uncommon, the treatment has large functional and oncological implications for the patient. Half of the tumours of the proximal fibula are malignant and require wide local excision. The proximal fibula is in close proximity to many important neurovascular and musculoskeletal compartments. Malawer ${ }^{3}$ hypothesised that because of the anatomical peculiarities of the proximal fibula, tumours behave differently in this area. The proximal fibula, with multiple muscle attachments and thin cortices, allows early cortical extension and direct invasion of surrounding structures, rather than the usual pushing growth and respect of fascial boundaries of sarcomas in other areas of the body.

The results of resection in the past were poor, showing high rates of re-operation for contaminated margins, and local recurrence, poor prognosis and functional results. As a result many centres advised amputation for malignant lesions of the proximal fibula. ${ }^{4}$ The concept of performing limb salvage surgery was revisited in the 1980s and was pioneered by Pritchard et al., ${ }^{5,6}$ Rosen et al., ${ }^{7}$ Bacci and Campanacci et al., ${ }^{8}$ and Malawer and Zaleskie. ${ }^{9}$ All showed good functional outcomes and equivalent cure rates using newer limb salvage techniques. Malawer in $1984^{3}$ described the specific approach and technique for resection of tumours of the proximal tibia.

This paper presents the curative and functional results of our series of Malawer-type I marginal resections for large active or aggressive benign tumours and low-grade sarcomas, and Malawer type II wide intra-compartmental resections for high-grade bone sarcomas.

\section{Methods and materials}

A retrospective folder review was performed from January 1995 to January 2017. Fourteen patients who underwent limb salvage for a proximal fibula neoplasm were included. This group was then subdivided into those who had a type I Malawer resection for a large active or aggressive benign tumour or low-grade malignant bone sarcoma, and those who received a type II resection for a stage IIB or higher ${ }^{10}$ primary bone sarcoma (Figure 1). Exclusion criteria included incomplete records and patients who were lost to follow-up. Two patients were excluded.

MRI (Figure 2) or CT was used to assess the lesion, patency of the relevant vasculature and position of the common peroneal nerve. CT angiography was used in selected cases if assessment of the vasculature was equivocal or inadequate on the MRI. Diagnosis was confirmed with open or 14-gauge needle biopsy in the operative theatre. For the stage IIB type II resections, patients received neo-adjuvant chemotherapy and no pre-operative radiotherapy. Adjuvant chemotherapy was commenced three to six weeks post-operatively. ${ }^{7}$ The patients who had type I resections received neither chemotherapy nor radiotherapy.

Follow-up was performed by the oncologists for the highgrade malignant lesions and included a chest X-ray and clinical examination every three months for the first two years, six monthly for years 2-5 and annually for years 5-10, at which point the patient was discharged. The patients with aggressive benign lesions or lowgrade malignant sarcomas were followed up by the orthopaedic surgeons with clinical examination and X-ray of the lesion every six months until discharge at two years. A Musculoskeletal Tumour Society (MSTS) score ${ }^{11}$ was performed at final follow-up.

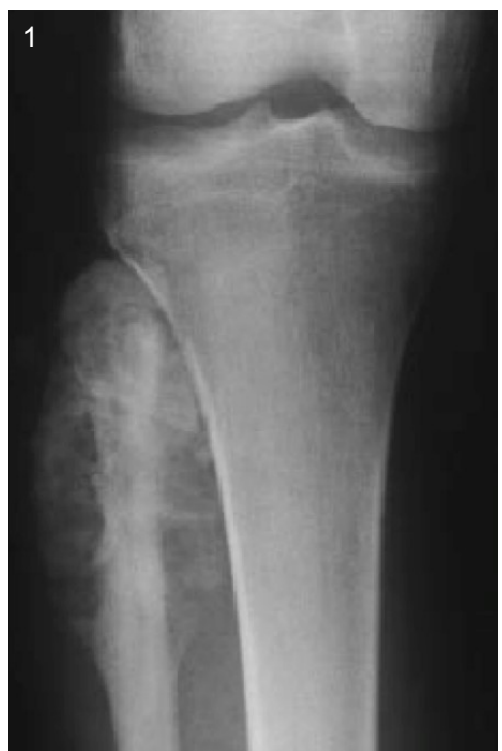

Figure 1. Antero-posterior X-ray of the proximal lower leg of a 12 -year-old female patient with an osteoblastic osteosarcoma of the proximal fibula after neoadjuvant chemotherapy

Figure 2. T2-weighted MRI pictures, coronal (a), sagittal (b) and axial (c), with a proximal fibula high-grade sarcoma

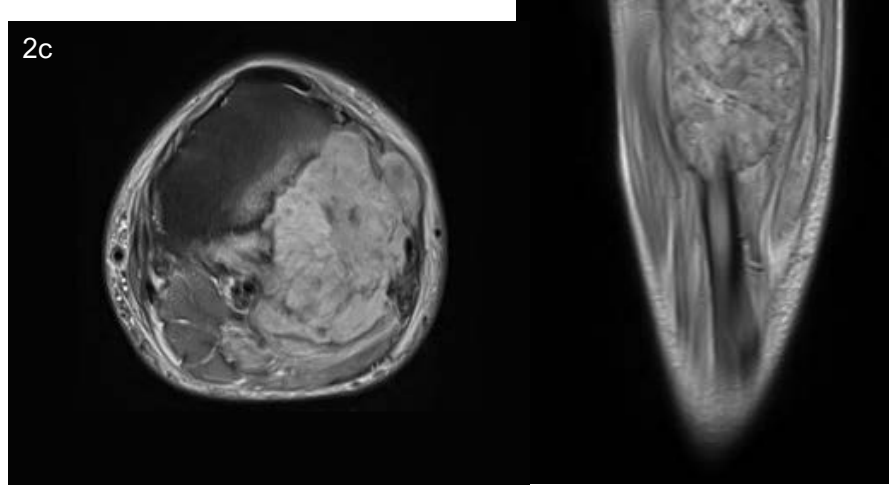

At a median follow-up of $38(6-123)$ months the clinical notes including the MSTS score, imaging and histology were reviewed. Descriptive statistical methods are used to present our findings. No further statistical methods were deemed necessary as no comparisons are being made and patient numbers are small.

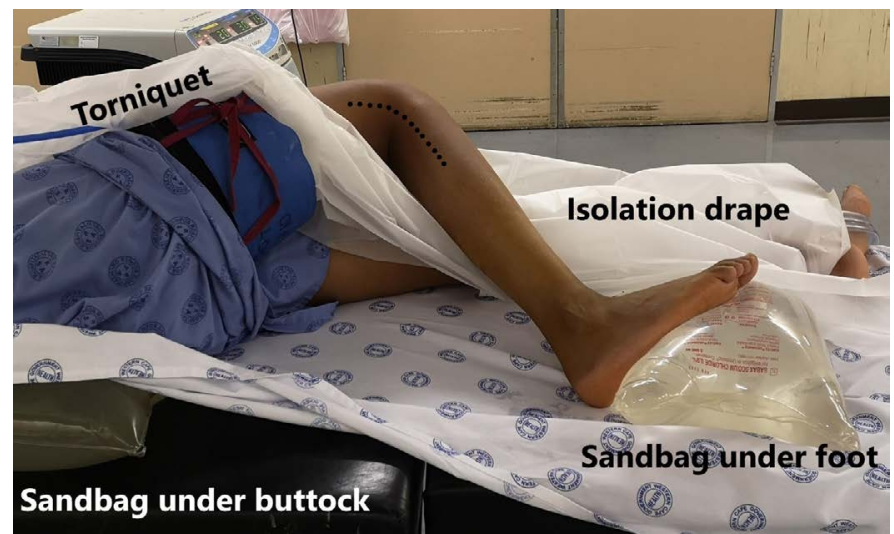

Figure 3. Illustration showing patient positioning and utilitarian incision with biopsy scar included for both type I and II resections 


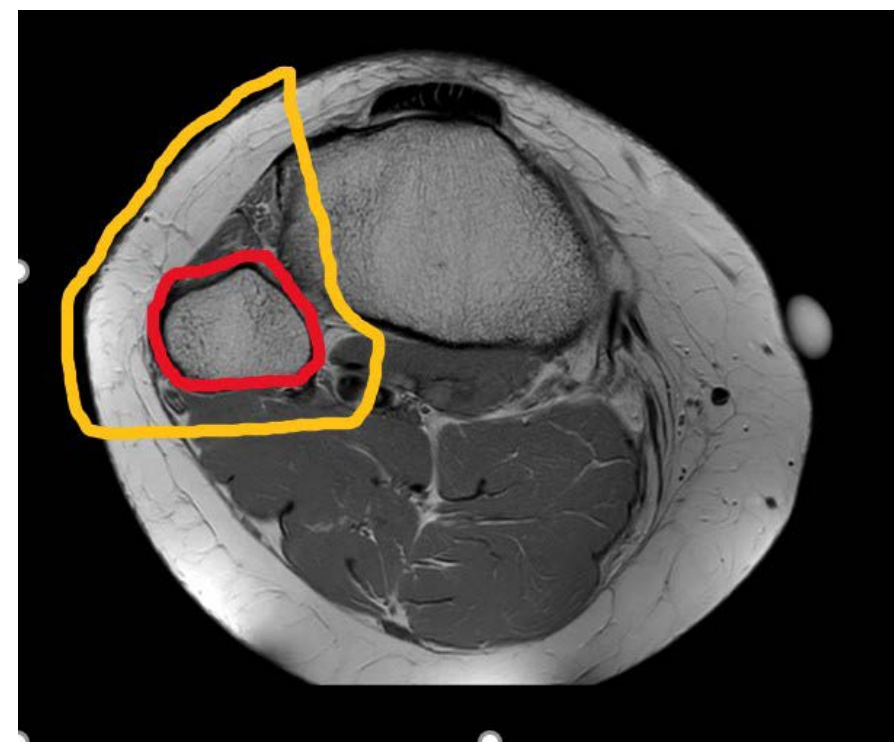

Figure 4. Axial MRI (T1-weighted) depicting the difference between type I (red) and type II (yellow) resections. Note type I resections include preservation of peroneal nerve, anterior, lateral and deep posterior muscular compartments with resection through the tibio-fibular joint. Type II resections sacrifice the anterior tibial artery, proximal portions of tibialis anterior, extensor hallucis longus, extensor digitorum longus, the peroneii muscles, FHL and TP muscles and include a shark-bite resection of the lateral cortex of the proximal tibia. The peroneal artery and nerve are resected as required.

\section{Surgical technique}

The surgical technique for en bloc resection of the proximal fibula was performed as described by Malawer in $1984 .^{3}$ A marginal excision of the proximal fibula is described as a type I and a wide intra-compartmental resection as a type II resection.

The patient is placed in a semi-supine position, with a sandbag under the ipsilateral buttock. This allows access to the anterior and lateral compartments, the popliteal space and the posterior compartment when the knee is flexed. ${ }^{3}$ It is prudent to prepare the limb for an above-knee amputation in the event that resection is not possible with a large and aggressive lesion. A single utilitarian anterolateral incision is used which includes the biopsy site and tract with an ellipse of surrounding skin. Placement of the biopsy site is therefore crucial (Figure 3).

Type I resections include the proximal fibula with $2-3 \mathrm{~cm}$ of normal diaphysis and a thin cuff of muscle in all dimensions (Figure 4 - red marker). The peroneal nerve and motor branches are preserved. The anterior tibial artery is sacrificed if necessitated by tumour extent. The artery is sacrificed in type II resections, but may be preserved in type I resections. The tibiofibular joint is excised intraarticularly. Reconstruction of the lateral collateral ligament (LCL) and biceps femoris tendon (BT) is to the proximal tibia, remaining soft tissue or anterolateral capsule, with the knee flexed at $30^{\circ}$. This may be achieved either with drill holes or the use of a bone anchor.

Type II resections (Figure 4 - yellow marker) include the proximal fibula and $6-7 \mathrm{~cm}$ of normal diaphysis, the proximal anterior and lateral muscle compartments, the proximal flexor hallucis longus (FHL) and tibialis posterior (TP) muscles, the anterior tibial artery, the peroneal nerve, and if required, the peroneal artery. The tibiofibular joint is resected extra-articularly with resection of the lateral tibial cortex and a variable portion of its metaphysis. Soft tissue reconstruction involves fixing the $L C L$ and $B T$ to the proximal tibia. The lateral gastrocnemius muscle is rotated to cover the surgical defect and exposed tibia. The muscle is released from the medial belly in the midline and distally as it joins the soleus.

Post-operative rehabilitation included three weeks of immobilisation at $30^{\circ}$ of flexion. Full weight bearing was allowed as tolerated. An ankle-foot orthosis was prescribed for type II resections if required. Some authors ${ }^{2,12}$ use a knee immobiliser for six weeks, during which motion exercises are started in the last two weeks.

\section{Results}

Six patients had a type I resection and eight a type II resection. The median follow-up period was 38 (6-123) months overall. The median follow-up for type I resections was 20 months and for type II was 47.5 months. Both groups had no recurrences or metastases. There were no wound or vascular complications. The characteristics of the patients and the MSTS score at follow-up are shown in Tables I and II.

Table I: Patients with type I resection

\begin{tabular}{|c|c|c|c|c|}
\hline Patient & Sex & Age at time of surgery (years) & Histology \\
\hline 1 & Male & 32 & Chondroblastoma \\
\hline 2 & Female & 53 & Chondrosarcoma \\
\hline 3 & Female & 60 & GCT \\
\hline 4 & Male & 53 & GCT \\
\hline 5 & Male & 17 & GCT \\
\hline 6 & Female & 14 & Osteochondroma \\
\hline
\end{tabular}

Table II: Patients with type II resection

\begin{tabular}{|c|c|c|c|c|c|}
\hline Patient & Sex & Age at time of surgery (years) & Histology & Huvos score & MSTS score \\
\hline 1 & Female & 13 & Osteoblastic osteosarcoma & $90 \%$ & 25 \\
\hline 2 & Male & 10 & Osteoblastic osteosarcoma & $100 \%$ & 24 \\
\hline 3 & Male & 6 & Ewing's sarcoma & $100 \%$ & 26 \\
\hline 4 & Male & 18 & Osteoblastic osteosarcoma & $25 \%$ & 26 \\
\hline 5 & Female & 83 & Leiomyosarcoma & $60 \%$ & 24 \\
\hline 6 & Female & 11 & Osteoblastic osteosarcoma & $0 \%$ & 30 \\
\hline 7 & Male & 11 & Osteoblastic osteosarcoma & $95 \%$ & 27 \\
\hline 8 & Male & 18 & Osteoblastic osteosarcoma & $95 \%$ & 27 \\
\hline
\end{tabular}




\section{Type I}

The median age for type I resections was $42.5(14-60)$ years. The commonest tumour was giant cell tumour (GCT) in three patients (50\%) and one each of an osteochondroma, chondrosarcoma and chondroblastoma.

The median MSTS functional score was 29 (15-30). No patients in the type I resection group required further surgical intervention.

\section{Type II}

The median age for type II resections was 12 (6-83) years, with seven of the eight patients being under 20 years of age at presentation. All the tumours were stage IIB. Osteoblastic osteosarcoma was the most common tumour (75\%). The remaining two patients were Ewing's sarcoma and leiomyosarcoma.

The median Huvos score was $92.5 \%$ (0-100\%). Five patients achieved $\geq 90 \%$ necrosis of the resected tumour after neo-adjuvant chemotherapy, two of which demonstrated $100 \%$ necrosis. The remaining three patients were alive without disease at their last follow-up (range 35-96 months). All patients achieved clear margins at initial surgery, and none required a secondary oncological procedure.

All patients reported good MSTS functional scores despite resection of the common peroneal nerve in all patients. The median score was 26 (24-30). One patient required a tibialis posterior tendon transfer (TPTT) for the management of an equinovarus foot following resection. His MSTS score post-TPTT was 26 . Of the remaining seven patients, six required an AFO.

\section{Discussion}

The more common Enneking sub-types of neoplasms found in this location include benign aggressive and malignant stage IIB lesions. Dahlin ${ }^{13}$ noted that the most common tumours in this location include GCT, osteogenic osteosarcoma and Ewing's sarcoma. In our study, similar to that of Abdel et al. ${ }^{2}$ and Dahlin, ${ }^{13}$ GCT was the commonest tumour (50\%) and osteogenic osteosarcoma the commonest tumour (75\%) undergoing type I and II resections respectively. For this reason and due to the crowded anatomy of the region, above-knee amputations were advised as the best oncological surgical option before $1980 .{ }^{4}$ However, with the onset of limb-sparing surgery the anatomy and resection techniques were revisited. ${ }^{3,5-9}$ The main concerns associated with proximal fibula resections are recurrence, post-operative knee instability, peroneal nerve palsy, and vascular insufficiency. ${ }^{2}$

The most important prerequisite of limb salvage is that the patient must be left with a limb of better or equal function than that of an amputation. ${ }^{14}$ In resections of the proximal fibula, ligation of two of the three major vessels, which is required in a type II resection, is compatible with a functional extremity, even in elderly patients with peripheral vascular disease.,15 Previously biplanar angiography was recommended by Hudson et al. ${ }^{16}$, and Marcove and Jensen. ${ }^{17} \mathrm{MRI}$ is currently used to identify the involvement, location of displaced vessels and the aberrant lack of the posterior tibial artery, which can occur in approximately $5 \%$ of cases. ${ }^{3}$ In the scenario of an absent posterior tibial artery, an above-knee amputation would be indicated. Other contra-indications to limb salvage include extension of the tumour intra-articularly into the knee and direct tibial involvement.

We had no vascular complications. This may be because only one of our patients was over 20 years of age (83 years).

Limb salvage must not compromise oncological outcome, as margins are all important for prognosis in high-grade malignant sarcomas of bone. An involved margin at resection increases the morbidity for the patient necessitating re-excision, possible amputation or post-operative radiotherapy. The attainment of a clear margin in resections of the proximal fibula is not straightforward. Malawer ${ }^{3}$ describes the direct invasion and serpiginous spread of stage IIB tumours along the multiple musculotendinous junctions of the surrounding muscles that originate off the fibular head and proximal shaft. This is in contrast to the pushing border that is more commonly seen with IIB tumours in other sites of the body. This may be due to the lack of fascial containment in this area and the relatively thin cortex of the fibula. The close proximity of the tibiofibular joint to the origin of these tumours leads to invasion of the tibiofibular joint by the aggressive sarcomas. This joint, along with its capsule and adjacent tibial cortex, must be included in the type II resections to attain a wide margin.

In the study by Abdel et al. ${ }^{2}$ local recurrence occurred in three of 24 patients (13\%) who had a type II resection and the postoperative rate of metastases was $50 \%$ for all 112 patients. Our study had a $100 \%$ negative margin rate in all high-grade tumours with no recurrences. We attribute this oncological success to ensuring adequate margins and by not attempting to preserve the common peroneal nerve where margins are doubtful.

Abdel et al. ${ }^{2}$ also report a high local recurrence rate in six of 29 (21\%) patients who had a type I resection for malignant tumours, especially osteosarcoma and chondrosarcoma. Although they do not note the Enneking staging ${ }^{10}$ for each tumour type, they recommend a type II resection for osteosarcoma and chondrosarcoma. We agree that a high-grade sarcoma with an extraosseous component warrants a type II resection, but for a low-grade intra-osseous chondrosarcoma, a type I resection is adequate.

In benign aggressive tumours intralesional curettage has a recurrence rate of $50-100 \%,{ }^{12,18}$ which supports a type I resection for these tumours. This differs from Enneking ${ }^{10,11}$ who advises intralesional curettage with an adjuvant with or without bone graft. The better results with a type I resection may be due to the unique anatomy of this area.

The Huvos scoring system evaluates the histology of the tumour post resection by grading the extent of necrosis relative to the percentage of residual viable tumour. The scoring system has been shown to be useful in prognosis and in the adjuvant chemotherapeutic management of both osteogenic osteosarcoma and Ewing's sarcoma. ${ }^{7}$ In our series of type II resections, $62.5 \%(n=5)$ of the patients achieved more than $90 \%$ necrosis, which, together with negative margins, contributed to their good outcomes. The remaining three patients in this group were alive without disease at their last follow-up. Their follow-up periods were 35, 47 and 96 months respectively.

Despite the sacrifice of the common peroneal nerve, all the patients who underwent type II resection reported a good functional outcome. Inatani et al. ${ }^{18}$ reiterate this with their report of a mean MSTS score of $20(65 \%)$ with sacrifice of the common peroneal nerve. In our series of type II resections the median MSTS score was 26 . Naturally in our type I resections, where a wide margin can be taken safely with preservation of the nerve, the median functional score improves to 29 .

Only one patient required a TPTT for the management of an equinovarus foot. The patient had a good functional outcome with a MSTS score of 27 . None of the other type II resection patients underwent this procedure and had equivalent scores, although six of the seven patients required an ankle-foot orthosis (AFO).

Peroneal nerve palsy has been described as a complication despite attempts at nerve preservation. In the paper by Abdel et al. ${ }^{2}$, two of $29(7 \%)$ patients suffered a palsy where the peroneal nerve was spared during a type I resection. The incidence in the literature varies between $20 \%$ and $57 \%$., $3,7,14$

Type I and type II resections include sacrificing adjacent knee 
stabilising structures. Unlike traumatic disruption of the lateral ligamentous structures, their reconstruction is controversial. Reconstruction of the biceps femoris tendon (BT) and lateral collateral ligament $(\mathrm{LCL})$ is performed by some authors; however, Einoder et al. ${ }^{19}$ believe that the reconstruction of the transected tendon and ligament is unnecessary as the integrity of the posterior capsule and other supporting structures such as the cruciate ligaments still remains. In their study however, the patients remained in a hinged knee brace for three months. Other authors $2,12,18$ advised BT and LCL reconstruction. In two of these studies, 2,12 83 knees were examined with a varus stress test at $30^{\circ}$ of knee flexion. No patient had more than grade 2 instability. We did not examine our patients formally with a varus stress test but no patient subjectively complained of instability.

There were no wound complications found in this study. Wound dehiscence, haematoma and flap necrosis can be prevented with a primary lateral gastrocnemius flap in type II resections. The rotated gastrocnemius belly also fills the defect created by resection of the proximal anterolateral muscles and fibula, and results in a more cosmetically acceptable shaped leg. Unfortunately, this causes delay in post-operative adjuvant oncological treatment, as the surgical wound requires time to heal before treatment is given Synovial fistula was not seen in our study and was seen in only one of ten patients in Malawer's ${ }^{3}$ original study. This complication can be prevented by meticulous closure of the posterior capsule and immobilising the knee in $30^{\circ}$ of flexion for three weeks.

This study is subject to the bias of a retrospective study. We had small numbers. It must be noted that this is a rare group of tumours and a smaller subset has been selected. Even in Abdel's paper $^{2}$, which spanned 97 years, they amassed only 112 patients with malignant tumours, of whom less than half $(n=53)$ underwent limb-sparing surgery and even fewer $(n=24)$ type II resections. We did not assess knee instability formally.

\section{Conclusions}

To achieve a favourable outcome, the biopsy technique is important and must be carried out to involve only one compartment; usually anterolaterally within the utilitarian incision to allow a 2-3 cm ellipse of skin with its tract to be included in the excision. Close but safe margins are achievable with the correct technique, and vital structures are retained as much as possible.

Our recommendations when faced with a lesion in the proximal fibula would depend on its histological diagnosis and Enneking staging..$^{10}$ Benign latent lesions should be managed conservatively with observation, while benign active lesions should be addressed with intra-lesional resection. A very large active lesion, such as the osteochondroma in our study, may require a type I Malawer resection. Benign aggressive lesions, such as GCTs, and malignant tumours without extra-osseous spread, such as the chondrosarcoma in our study, should be excised en bloc using the type I Malawer resection. All malignant stage IIB tumours, i.e. with extra-osseous extension, should be treated with a type II Malawer resection with no attempt to spare the common peroneal nerve. This is to ensure a good oncological result with a functionally acceptable limb.

Resection of malignant and benign tumours of the proximal fibula in this series achieved good cure rates and functional results, with or without the aid of an orthosis, despite the sacrifice of the common peroneal nerve in type II resections.

\section{Ethics statement}

This study was subject to an institutional review by the Human Research Ethics Committee number $807 / 2017$.

\section{Declaration}

The authors declare authorship of this article and that they have followed sound scientific research practice. This research is original and does not transgress plagiarism policies.

\section{Author contributions}

TLH - ethics, data collection, analysis, write up; KW - write up, analysis; KVH proposal, data collection; EBH - data collection, final write up.

\section{ORCID}

Hilton TL (D) https://orcid.org/0000-0002-6178-5062

Wiese KR iD https://orcid.org/0000-0003-1764-8500

Hosking KV (iD https://orcid.org/0000-0002-3557-0252

Hoffman EB iD https://orcid.org/0000-0001-5954-0069

\section{References}

1. Unni KK. Dahlin's bone tumors: general aspects and data on 11,087 cases. Philadelphia: Lippincott Williams \& Wilkins, 1996. 1-9

2. Abdel MP, Papagelopoulos $P$, Morrey ME, Inwards CY, Wenger $\mathrm{DE}$, Rose DE, Sim FH. Malignant proximal fibular tumors: surgical management of 112 cases. J Bone Joint Surg Am, 2012;94(22):165.

3. Malawer MM. Surgical management of aggressive and malignant tumors of the proximal fibula. Clin Orthop Relat Res, 1984(186):172-81.

4. Malawer M, Sugarbaker PH. Fibular Resection. In: Musculoskeletal Cancer Surgery Treatment of Sarcomas and Allied Diseases. Kluwer Academic Publishers, 2001. 503-16.

5. Pritchard DJ, Dahlin DC, Dauphine RT, Taylor WF, Beabout JW. Ewing's sarcoma. A clinicopathological and statistical analysis of patients surviving five years or longer. $J$ Bone Joint Surg Am, 1975;57(1):10-16.

6. Pritchard D. Ewing's sarcoma. Presented to the musculoskeletal Tumour Society, New York, April 1982.

7. Rosen G, Caparros G, Nirenberg A, Ralph C, Marcove MD, Huvos $A G$, et al. Ewing's sarcoma: ten-year experience with adjuvant chemotherapy. Cancer, 1981;47(9):2204-13.

8. Bacci G, Picci P, Gitelis S, Borghi A, Campanacci M. The treatment of localized Ewing's sarcoma: the experience at the Istituto Ortopedico Rizzoli in 163 cases treated with and without adjuvant chemotherapy. Cancer, 1982;49(8):1561-70.

9. Malawer MM, Zaleskie T. Giant cell tumour of bone: En-bloc resection and cryosurgery: Evaluation, indications and recommendations for treatment. Orthop Trans, 1982;6:91.

10. Enneking WF, Spanier SS, Goodman MA. A system for the surgical staging of musculoskeletal sarcoma. Clin Orthop Relat Res, 1980;153:106-20.

11. Enneking WF, A system of staging musculoskeletal neoplasms. Clin Orthop Relat Res, 1986;204:9-24.

12. Abdel MP, Papagelopoulos PJ, Morrey ME, Wenger DE, Rose PS, Sim FH. Surgical management of 121 benign proximal fibula tumors. Clin Orthop Relat Res, 2010;468(11):3056-62.

13. Dahlin DC. Bone tumours, general aspects and data on 6,221 cases. Springfield, 1978. 11-12.

14. DiCaprio MR, Friedlaender GE. Malignant bone tumors: limb sparing versus amputation. J Am Acad Orthop Surg, 2003;11(1):25-37.

15. Imparato AM, Roses DF, Francis KC, Lewis MM. Major vascular reconstruction for limb salvage in patients with soft tissue and skeletal sarcomas of the extremities. Surg Gynecol Obstet, 1978;147(6):891-96.

16. Hudson TM, Haas G, Enneking WF, Hawkins IF. Angiography in the management of musculoskeletal tumors. Surg Gynecol Obstet, 1975;141(1):11-21.

17. Marcove RC, Jensen MJ. Radical resection for osteogenic sarcoma of fibula with preservation of the limb. Clin Orthop Relat Res, 1977;125:173-76.

18. Inatani $\mathrm{H}$, Yamamoto $\mathrm{N}$, Hayashi $\mathrm{K}$, Kimura $\mathrm{H}$, Takeuchi A, Miwa $\mathrm{S}$, et al. Surgical management of proximal fibular tumors: A report of 12 cases. J Bone Oncol, 2016;5(4):163-66.

19. Einoder PA, Choong PF. Tumors of the head of the fibula: good function after resection without ligament reconstruction in 6 patients. Acta Orthop Scand, 2002;73(6):663-66. 